\title{
Small intestine bacterial overgrowth is frequent in cystic fibrosis: combined hydrogen and methane measurements are required for its detection
}

\author{
Aleksandra Lisowska ${ }^{1}$, Jerzy Wójtowicz ${ }^{2}$ and Jarosław Walkowiak ${ }^{1 \bowtie}$ \\ ${ }^{1}$ Department of Pediatric Gastroenterology and Metabolism, and ${ }^{2}$ Department of Otolaryngology, \\ Poznań University of Medical Sciences, Poznań, Poland
}

Received: 15 April, 2009; 16 November, 2009; accepted: 08 December, 2009

available on-line: 09 December, 2009

\begin{abstract}
Introduction: Hydrogen breath test (BT) is commonly used as a diagnostic tool for the detection of small intestine bacterial overgrowth (SIBO). It was reported that colonic methane production is far more frequent in cystic fibrosis (CF) patients than in other subjects. Therefore, measuring exclusively hydrogen in the diagnostic breath test for diagnosing SIBO might be of limited value. We aimed to assess the usefulness of combined measurement of hydrogen and methane expiration for the diagnosis of SIBO in CF. Material and Methods: The study comprised 62 CF patients aged 5 to 18 years. Three-hundred-ninety subjects assessed due to gastrointestinal symptoms for the presence of SIBO served as a comparative group. In all subjects hydrogen/methane BT using glucose was performed. A positive BT was defined as fasting hydrogen $\geq 20 \mathrm{ppm}$ or fasting methane $\geq 10 \mathrm{ppm}$ or a rise of $\geq 12 \mathrm{ppm}$ hydrogen or $\geq 6 \mathrm{ppm}$ methane over baseline during the test. Results: In $23(37.1 \%)$ CF patients and in $52(13.3 \%)$ subjects from the comparative group abnormal BT results were found. In seven $(11.3 \%) \mathrm{CF}$ patients and $29(7.4 \%)$ of the other subjects studied methane measurement allowed diagnosis of SIBO. Conclusions: Small intestine bacterial overgrowth is frequent in cystic fibrosis. For its detection in cystic fibrosis and other gastrointestinal patients, combined hydrogen and methane measurement instead of hydrogen breath test should be applied. Without the additional measurement of methane a significant percentage of SIBO will be missed.
\end{abstract}

Keywords: cystic fibrosis, small intestine bacterial overgrowth, breath test, hydrogen, methane

\section{INTRODUCTION}

It has been reported that small intestine bacterial overgrowth (SIBO) occurs in $30-50 \%$ of cystic fibrosis (CF) patients (Lewindon et al., 1998; Fridge et al., 2007). Bacterial colonization of the upper part of the intestine, may significantly influence the function and structure of digestive system in CF (Borowitz et al., 2005). Therefore, all patients should be routinely tested for its presence.

Hydrogen breath test (BT) with glucose loading is commonly used as a diagnostic tool for the detection of SIBO (Singh \& Toskes, 2003; Husebye, 2005). It was demonstrated in non-CF subjects, however, that small intestine flora is also capable of producing methane (Perman \& Modler, 1982). So far, there are no data related to the limitations of exclusive measurement of hydrogen as a diagnostic procedure for SIBO detection in CF. The colonic flora in CF patients is far more frequently not capable of producing hydrogen from non-digestable lactulose compared to controls (Lewindon et al., 1998) and also methane production is far more frequent in CF patients than in other subjects (Bujanover et al., 1989). Therefore, we hypothesized that in a significant percentage of CF patients with SIBO, small intestine methane production may appear. The aim of the present study was to assess the usefulness of combined measurement of hydrogen and methane expiration for the diagnosis of SIBO in CF.

\footnotetext{
${ }^{\square}$ Corresponding author: Jarosław Walkowiak, 1st Chair of Pediatrics, Department of Gastroenterology \& Metabolism, Poznań University of Medical Sciences, Szpitalna 27/33, 60-572 Poznań, Poland; Tel.: (48) 61 848 0310; E-mail jarwalk@ am.poznan.pl

Abbreviations: BT, breath test; CF, cystic fibrosis; GI, gastrointestinal; GS, gastrointestinal symptoms; P.O. per os; SIBO, small intestine bacterial overgrowth.
} 


\section{MATERIAL AND METHODS}

Subjects. The study comprised $62 \mathrm{CF}$ patients aged 5 to 17 years. The diagnosis of $\mathrm{CF}$ was made upon history, clinical manifestation, increased sweat chloride concentrations (two independent measurements) and confirmed by the CFTR gene analysis.

Inclusion criteria for CF patients comprised the ability and willingness to participate in the study and pancreatic insufficiency. Exclusion criteria in the CF group comprised: antibiotic therapy (i.v. or per os) six weeks prior to the test, PPI and steroid use, liver cirrhosis, diabetes mellitus, oxygen dependency and pulmonary exacerbation within three preceding months. None of the patients reported major abdominal complaints (unusual for her and/or his typical manifestation). Three-hundred-ninety subjects referred, due to gastrointestinal symptoms, for the BT to exclude SIBO served as a comparative group. Inclusion criteria for this group comprised the ability and willingness to participate in the study. Exclusion criteria comprised antibiotic therapy (i.v. or PO) six weeks prior to the test. In all subjects hydrogen/ methane BT with glucose loading was performed.

Methods. All patients underwent glucose BT after overnight fasting. Each of them received glucose dissolved in water orally in a dose $1.5 \mathrm{~g} / \mathrm{kg}$ up to maximum $75 \mathrm{~g}$. Breath samples were collected at baseline (fasting) and at 15, 30, 45, 60, 90, $120 \mathrm{~min}$ after glucose ingestion. Samples were analyzed with QuinTron MicroLyser DP Plus (Quintron, USA).

A positive BT was defined as fasting hydrogen $\geq 20 \mathrm{ppm}$ or fasting methane $\geq 10 \mathrm{ppm}$ or a rise of $\geq 12 \mathrm{ppm}$ hydrogen or $\geq 6 \mathrm{ppm}$ methane over baseline during the test.

Ethical considerations. The protocol of the investigation has been approved by the Ethical Committee of the Poznań University of Medical Sciences (Poland).

\section{RESULTS}

The genotypes of CF patients studied were as follows: F508del/F508del $(\mathrm{n}=32), \quad$ F508del/ CFTRdel2,3(21kB) $\quad(\mathrm{n}=4), \quad$ F508del/R553X $\quad(\mathrm{n}=1)$, F508del/2143delT $\quad(n=1), \quad$ CFTRdel2,3(21kB)/ CFTRdel2,3(21kB) $(\mathrm{n}=3)$, CFTRdel2,3(21kB)/2143delT $(\mathrm{n}=1), \mathrm{F} 508 \mathrm{del} /$ unknown mutation $(\mathrm{n}=12)$, unknown mutation/unknown mutation $(n=6)$. The basic clinical characteristics of the CF patients are presented in Table 1 .

In $23(37.1 \%)$ CF patients and in $52(13.3 \%)$ subjects from the comparative group abnormal BT results were found. SIBO was significantly more frequent in the CF patients with mild/no manifesta-
Table 1. Basic clinical data of CF patients studied

\begin{tabular}{ll}
\hline Parameter & (median/mean \pm S.E.M.) \\
\hline Z-score for body weight & $-0.95(-0.98 \pm 0.12)$ \\
FEV1 $(\%)$ & $73(77 \pm 4)$ \\
Fecal elastase-1 $(\mu \mathrm{g} / \mathrm{g}$ of stool) & $9(26 \pm 3)$ \\
\hline
\end{tabular}

tion than in the non-CF subjects $(P<0.00001)$. The distribution of normal and abnormal hydrogen and methane expiration in subjects studied is presented in Table 2. In eleven (17.7\%) CF patients and 29 (7.4\%) of the other subjects studied methane production allowed for the diagnosis of SIBO.

In seven $(11.3 \%)$ patients with documented methane production hydrogen expiration was low (less than $5 \mathrm{ppm}$ ). Therefore, methane measurement led to the diagnosis of SIBO in $30.4 \%$ (7 out of 23 ) of the detected cases.

\section{DISCUSSION}

In the present study we have proven that small intestine bacterial overgrowth in CF is frequent and that combined hydrogen and methane measurement improves the sensitivity of the breath test. In the comparative group of subjects presenting gastrointestinal symptoms the occurrence of SIBO was less common. The percentage of potential false negative results due to exclusive hydrogen assessment was, however, not different.

Bujanover et al. (1987) assessed the frequency of methane production in $28 \mathrm{CF}$ patients and 290 age-matched healthy subjects (HS). Seventeen $(60.7 \%)$ CF and 60 (20.6\%) HS were found to produce methane. Mean gas concentrations in the methane-producers were rather low $(6.5$ and $6.0 \mathrm{ppm}$ in $\mathrm{CF}$ and HS, respectively; the range of values in $\mathrm{CF}$ patients: 1-15.8 ppm). The subjects were, however, non-fasted, eating earlier non-standardized meals (with unknown intake of different sugars). Therefore, the site of gas production remains unclear. This renders a direct comparison of the values reported by Bujanover with those obtained in standard BTs impossible. In addition, the majority of the subjects in Bujanovers's study had high fecal fat losses (from 4.7 to $65 \mathrm{~g} /$ day) pointing to malabsorption. There-

Table 2. Distribution of normal and abnormal hydrogen and methane expiration in cystic fibrosis (CF) patients and subjects presenting gastrointestinal symptoms (GS)

\begin{tabular}{llllll}
\hline & \multicolumn{3}{l}{ Breath test results } & \multirow{2}{*}{$P$} \\
\cline { 2 - 5 } & CF & \multicolumn{3}{l}{ GS } & \\
\cline { 2 - 5 } & Abnormal & Normal & Abnormal & Normal & \\
\hline Hydrogen & 12 & 50 & 23 & 367 & 0.0002 \\
Methane & 11 & 51 & 29 & 361 & 0.008 \\
\hline
\end{tabular}


fore, the measured methane production might have reflected malabsorption rather than small intestine bacterial overgrowth. The significance of detectable methane production in non-fasted CF subjects for the detection of potential abnormalities remains to be elucidated.

Lewindon et al. (1998) retrospectively analyzed the prevalence of carbohydrate malabsorption and SIBO in a CF population. Results from 89 hydrogen BTs with lactose $(n=43)$, sucrose $(n=15)$ and lactulose $(n=31)$ performed in 54 CF subjects were compared with the results of available BTs done in non-CF patients $(n=5430)$. The mean age of the CF children (30.2 months; range 1-212 months) was not different from that of the non-CF population (29.4 months; range 1-228 months). CF patients were investigated due to abnormal stool pattern. All CF patients were taking antibiotics (either for therapeutic or prophylactic purposes). The most common indications for BT in the non-CF population were as follows: lactose BT-suspected post-eneteritis lactose malabsorption, chronic diarrhoea and suspected constitutional lactase deficiency, sucrose BT-suspected severe post-enteritis carbohydrate malabsorption, chronic diarrhoea and suspected sucrase-isomaltase deficiency, lactulose BT-suspected bacterial overgrowth, suspected post-antibiotic inability of GI flora to produce hydrogen. Additionally, the results of lactulose BT from 22 healthy children were available for comparison, their age being, however, differed (mean 43.6 months; range 5-108 months). Taking into account the differences between the groups one should have in mind the limitations of such comparison. Nevertheless, CF patients more frequently were unable to produce hydrogen from lactulose as compared to non-CF patients and HS $(39 \%, 20 \%$ and $5 \%$, respectively). More CF subjects than non-CF patients had SIBO (32\% vs. $7 \%)$. Due to the lack of methane detection, however, the role of its measurement for the diagnosis of SIBO was not established.

Increased fasting methane levels in patients with SIBO have been reported. It has been suggested that this finding is related to increased quantity of glycoproteins in intestine (Perman \& Modler, 1982). Abnormal mucins and SIBO are a hallmark of the CF mouse model (Clarke et al., 2004; Norkina et al., 2004). Therefore, one could expect higher production of methane in CF subjects than in the general population. In vitro, antibiotics are used to cultivate methanogenic flora (Miller et al., 1980). Since CF patients routinely undergo antibiotic therapy (either for therapeutic or prophylactic purposes) it could constitute an additional underlying "risk factor" for selecting methane-producing flora. None of our patients were chronically treated with azithromycin, which was suggested by Fridge et al. (2007) to be a risk factor for SIBO in CF. Therefore, methane production in other CF patients with SIBO could be even higher than in our studied population. Alveolar hydrogen concentrations are potentially suppressed by methanogenic bacteria (Perman \& Modler, 1982). Thus, with the use of hydrogen breath test one could get false negative results, on the one hand because methane is not measured, and on the other because hydrogen could be falsely low. All these points underline the need of methane measurement.

Interestingly, in the present study the percentage of methane-related positive BT results was not higher in CF patients than in non-CF subjects. As mentioned earlier the ability to produce methane seems to be higher in CF than in HS subjects (Bujanover et al., 1987). In a cross-sectional study of 1162 Polish children and adolescents aged 2 to 19 years the ability to produce methane was found in $24.8 \%$ of them (Kamińska et al., 2002). The general ability to produce methane by gastrointestinal bacteria is, however, not equivalent to methane production in the case of SIBO. Moreover, in the present study we compared CF patients not with HS but with subjects with gastrointestinal complaints having different intestinal "backgrounds" with potentially different intestinal flora and frequently being also previously treated with antibiotics.

The clinical significance of SIBO in CF patients without overt clinical manifestation is not clear. As opposed to animal models (Norkina, 2004), there are no published data documenting the influence of SIBO on maldigestion and nutritional status of CF patients and they should be elucidated. Taking into account the positive results of probiotic randomized control trial in CF (Bruzzesse et $a l ., 2007)$ not only on the gastrointestinal tract, their routine use should be considered. In an open study (Bruzzesse et al., 2004), decreased values of indirect indicators of intestinal inflammation have also been documented. Nevertheless, the influence of probiotics on the excessive bacterial colonization of small intestine in CF patients is not known, neither is there evidence in terms of specific bacterial colonization of small intestine due to SIBO in CF. It has been, however, suggested in non-selected patients with SIBO that the condition is neither related nor limited to any particular bacterial strain. Hydrogen BT has been commonly used as a diagnostic tool for the detection of SIBO. Although it has limited sensitivity and specificity (Kerckhoffs et al., 2008), it is the most reliable one of the available non-invasive tests. However, it has not routinely been used in CF patients. In the present study we hypothesized and have proved that in a significant percentage of CF patients with SIBO, small intestine methane production appears and allows for the detection of excessive bacterial colonization of the small intestine. A similar finding has been documented in 
non-CF subjects with gastrointestinal complaints. Not performing methane measurement would lead to a significant percentage of false negative results. Therefore, hydrogen-methane BT instead of hydrogen BT alone should be used for testing SIBO.

\section{CONCLUSIONS}

Small intestine bacterial overgrowth is frequent in cystic fibrosis. For its detection in cystic fibrosis and other gastrointestinal patients, combined hydrogen and methane measurement instead of hydrogen breath test should be applied. Without the additional measurement of methane a significant percentage of SIBO will be missed.

\section{Acknowledgements}

Supported by the Ministry of Science and Higher Education (grant No. N406 05431).

\section{REFERENCES}

Borowitz L, Durie PR, Clarke LL, Werlin SL, Taylor CJ, Semler J, De Lisle RC, Lewindon P, Lichtman SM, Sinaasappel M, Baker RD, Baker SS, Verkade HJ, Lowe ME, Stallings VA, Janghorbani M, Butler R, Heubi J (2005) Gastrointestinal outcomes and confounders in cystic fibrosis. J Pediatr Gastroenterol Nutr 41: 273-285.

Bruzzesse E, Raia V, Gaudiello G, Polito G, Buccigrossi V, Formicola V, Guarino A (2004) Intestinal inflammation is a frequent feature of cystic fibrosis and is reduced by probiotic administration. Aliment Pharamcol Ther 20: 813-819.

Bruzzesse E, Raia V, Spagnuolo MI, Volpicceli M, De Marco G, Maiuri L, Guarino A (2007) Effect of Lactobacil- lus GG supplementation on pulmonary exacerbations in patients with cystic fibrosis: a pilot study. Clin Nutr 26: 322-328.

Bujanover Y, Peled Y, Blau H, Yahav J, Katznelson D, Gilat T (1987) Methane production in patients with cystic fibrosis. J Pediatr Gastroenterol Nutr 6: 377-380.

Clarke LL, Gawenis LR, Bradford EM, Judd LM, Boyle KT, Simpson JE, Shull GE, Tanabe H, Ouellette AJ, Franklin CL, Walker NM (2004) Abnormal Paneth cell granule dissolution and compromised resistance to bacterial colonization in the intestine of CF mice. Am J Physiol Gastrointest Liver Physiol 286: 1050-1058.

Fridge JL, Conrad C, Gerson L, Castillo RO, Cox K (2007) Risk factors for small bowel bacterial overgrowth in cystic fibrosis. J Pediatr Gastroenterol Nutr 44: 212-218.

Husebye E (2005) The pathogenesis of gastrointestinal bacterial overgrowth. Chemiotherapy 51 (Suppl 1): 1-22.

Kamińska B, Landowski P, Bogotko M, Szarszewski A, Korzon M (2002) The incidence of breath methane population in children and youth from Gdańsk City. Pediatria Wspótczesna 4: 265-267 (in Polish).

Kerckhoffs AP, Visser MR, Samsom M, van der Rest ME, de Vogel J, Harmsen W, Akkermans LM (2008) Critical evaluation of diagnosing bacterial overgrowth in the proximal small intestine. J Clin Gastroenterol 42: 1095-1102.

Lewindon PJ, Robb TA, Moore DJ, Davidson GP, Martin AJ (1998) Bowel dysfunction in cystic fibrosis: importance of breath testing. J Pediatr Child Health 34: 79-82.

Miller TL, Wolin MJ (1982) Enumeration of methanobrevibacter Smithii in human feces. Arch Microbiol 131: 14-18.

Norkina O, Burnett DG, De Lisle RC (2004) Bacterial overgrowth in cystic fibrosis transmembrane conductance regulator null mouse small intestine. Infect Immun 72: 6040-6049.

Perman JA, Modler S (1982) Glycoproteins as substrates for production of hydrogen and methane by colonic bacterial flora. Gastroenterology 83: 388-393.

Singh VV, Toskes PP (2003) Small bacterial overgrowth: presentation, diagnosis and treatment. Curr Gastroenterol Rep 5: 365-372. 